\title{
Neutron Stars and the High Density Equation of State
}

\author{
T. Klähn*, C.D. Roberts*, D.B. Blaschke ${ }^{\dagger, * *}$ and F. Sandin \\ *Physics Division, Argonne National Laboratory, Argonne IL 60439, USA \\ $\dagger$ Institute for Theoretical Physics, University of Wroclaw, 50-204 Wroclaw, Poland \\ ** Bogoliubov Laboratory for Theoretical Physics, JINR Dubna, 141980 Dubna, Russia \\ ¥IFPA, Département AGO, Université de Liège, Sart Tilman, 4000 Liège, Belgium
}

\begin{abstract}
One of the key ingredients to understand the properties of neutrons star円 (NS) is the equation of state at finite densities far beyond nuclear saturation. Investigating the phase structure of quark matter that might be realized in the core of NS inspires theory and observation. We discuss recent results of our work to point out our view on challenges and possibilities in this evolving field by means of a few examples.
\end{abstract}

Keywords: neutron stars, nuclear matter, quark matter, equation of state, QCD phase transition PACS: $12.38 . L g$ 12.39.Ki 21.65.Qr 26.60.Kp 97.60.Jd

\section{INTRODUCTION}

Not only due to their compactness with central densities multiples beyond nuclear saturation NS are considered to belong to the most interesting systems nature offers to study the properties of ultra-dense matter. But it is this feature that provides a basis for the wide range of unique possibilities that NS offer to gain insight into the very nature of fundamental interactions. A fascinating and yet undecided question is whether the QCD phase transition from nucleonic to the fundamental quark matter (QM) degrees of freedom is realized in the core of NS. Answering this question in a strict sense would require the theorist to describe nuclear matter at finite densities in the framework of QCD, which is challenging already in vacuum. Solving QCD in medium at this fundamental level is a yet unsolved task even though promising work into this direction has been done. In a common approach to bypass this problem quark and nuclear matter EoS are modeled separately and are combined afterwards by constructing a phase transition thermodynamically. However, already pure quark or nuclear matter EoS are barely constrained due to the few reliable data at densities beyond saturation.

This paper embraces aspects of our recent work on ultra-dense matter. It starts with a discussion of potential promising constraints on the EoS of ultra-dense matter from NS mass and mass-radius measurements and the elliptic flow in heavy ion collisions. These, other constraints and more detailed discussions can be found in [2, 3, 4]]. The two following sections consider the EoS in nuclear and quark matter. Here we focus on the importance of constraining in particular the nuclear EoS [2] and discuss present approaches on describing quark matter in medium. While deconfinement and dynamical chiral symmetry breaking are accessible by solving QCD's gap equation within the framework of the Dyson-Schwinger formalism [5], effective models of the NJL-type are a practical tool to explore the phase structure of quark matter at finite densities [6, 7]. Finally, we discuss a recently introduced approach to construct the phase transition from nuclear to quark matter which results in a possibly mixed phase of one-flavor quark matter on a background of nuclear matter [7].

\section{CONSTRAINTS ON THE EQUATION OF STATE}

Far from the basic perception of a NS as an object as heavy, and heavier, as our sun, bound by gravity into a sphere with a diameter of about $25 \mathrm{~km}$ and spinning around its axis up to 1000 times per second (which is already fascinating) $N S$ are complex and inherently versatile objects. This is because NS have an internal structure and evolve in time. These two elements - structure and temporal history - are both a challenge and the key for a detailed understanding of

\footnotetext{
1 We use the generic term neutron stars for simplicity. In the context of this paper this does not exclude any exotic form of strongly interacting matter in the NS core such as color superconducting quark matter [1].
} 
the physics of matter in NS. With increasing observational efforts especially over the last decade, which will increase further over the next, NS data become more and more valuable as constraints for the modeling of ultra-dense matter. Of particular interest are NS which significantly deviate from the norm since those define the natural limits one has to account for in any reliable theoretical description.

Facing the present potential uncertainties in observation and modeling, it makes sense to start from an 'extremal set' of observables (largest and smallest known NS mass, radius, moment of inertia, rotational period, etc.) and to examine the consistency of this set, e.g. for similar underlying model assumptions. The benefit of this approach is apparent in two ways. First, a reliable EoS should be in agreement with all trusted NS data available. Second, taking into account extremal data is a valuable additional strategy to explore the real limits of NS properties and the EoS. Therefore, at present, the challenge is to identify both reliable "extreme" NS data and possible contradicting or complementing data that might narrow the limits of NS observables and the EoS.

\section{Neutron Star Masses}

For the purpose of this work we focus on NS configurations which are extreme in terms of their mass and/or radius. Due to observational uncertainties one should be careful not to mistake these extreme values as the final truth on the subject. A relativistic analysis of timing observations of the pulsar PSR J1903+0327 finds its mass to be $1.74 \pm 04 \mathrm{M}_{\odot}$. This is the largest pulsar mass that has ever been precisely measured [8]. Evidence for more massive NS exists but suffers from larger uncertainties. We consider a maximum NS mass of about $2.0 \mathrm{M}_{\odot}$, which is in agreement with timing measurements for PSR B1516+02B, located in the globular cluster M5. The derived pulsar mass is $(2.08 \pm 0.19) \mathrm{M}_{\odot}$ (at $68 \%$ probability) with a $95 \%$ probability that the mass of this object is above $1.72 \mathrm{M}_{\odot}$ [9] which deviates considerably from the average masses of binary radio pulsars, $\mathrm{M}_{\mathrm{BRP}}=1.35 \pm 0.04 \mathrm{M}_{\odot}$ [10]. However, looking at the recent history and the prominent example of PSR J0751+1807 we are aware of the fact that the identification of NS masses is a sensitive task and might give flawed results. In the latter case the mass initially determined to be $2.1 \pm 0.2 \mathrm{M}_{\odot}(1 \sigma)$ had to be corrected to a value of $1.26 \pm 0.2 \mathrm{M}_{\odot}(1 \sigma)$ due to new data analysis. There are other observations pointing towards high NS masses $\left(2.10 \pm 0.28 \mathrm{M}_{\odot}\right.$ for EXO 0748-676,2.0 $\pm 0.1 \mathrm{M}_{\odot}$ for 4U 1636-536) but for the given reason they should be treated with caution. As we will point out, the analysis of the elliptic flow in heavy ion collisions suggests that $2 \mathrm{M}_{\odot}$ is an approximate upper limit on NS masses, but presently no other criterion is known, that would decisively rule out the existence of high massive NS. It will depend on future observations to affirmatively determine the actual upper limit on NS masses.

\section{Radii and Mass-Radius Relations}

Aside from NS masses, kilohertz quasi-periodic brightness oscillations (QPOs) seen from more than 25 low-mass NS X-ray binaries (LMXBs) can be used to put additional constraints on the high-density EoS. A pair of such QPOs is often seen from these systems [11]. In all currently viable models for these QPOs, the higher QPO frequency is close to the orbital frequency at some special radius. For such a QPO to last the required many cycles (up to $\sim 1000$ in some sources), the orbit must be outside the star. According to general relativity theory the orbit must also be outside the innermost stable circular orbit (ISCO). Gas or particles inside the ISCO would spiral rapidly into the star, preventing the production of sharp QPOs. This implies [12,13] that the observation of a source whose maximum QPO frequency is $v_{\max } \approx v_{\mathrm{ISCO}}$ limits the stellar mass and radius to

$$
M<2.2 \mathrm{M}_{\odot} \frac{1000 \mathrm{~Hz}}{v_{\max }}(1+0.75 j), \quad R<19.5 \mathrm{~km} \frac{1000 \mathrm{~Hz}}{v_{\max }}(1+0.2 j) .
$$

The quantity $j \equiv c J / G M^{2}$ (with $J$ the stellar angular momentum) is the dimensionless spin parameter, which is typically in the range between 0.10 and 0.2 for these systems. Equation (1) implies that for given observed value $v_{\max }$ the mass and radius of that source must be inside a wedge-shaped area, as shown in Fig. 11 Since the wedge becomes smaller for higher $v_{\max }$, the highest frequency ever observed, $1330 \mathrm{~Hz}$ for $4 \mathrm{U} 0614+091$ [14], places the strongest constraint on the EoS. As can be seen from Fig. 11 the current QPO constraints do not rule out any of the EoS considered here. However, because higher frequencies imply smaller wedges, the future observation of a QPO with a frequency in the range of $\sim 1500-1600 \mathrm{~Hz}$ would rule out the stiffest of our EoS.

If there is evidence for a particular source that a given frequency is close to the orbital frequency at the ISCO, then the mass is known to a good accuracy, with uncertainties arising from the spin parameter. This was first claimed for 
4U 1820-30 [15], but complexities in the source phenomenology have made this controversial. More recently, careful analysis of Rossi X-ray Timing Explorer data for 4U 1636-536 and other sources [16] has suggested that sharp and

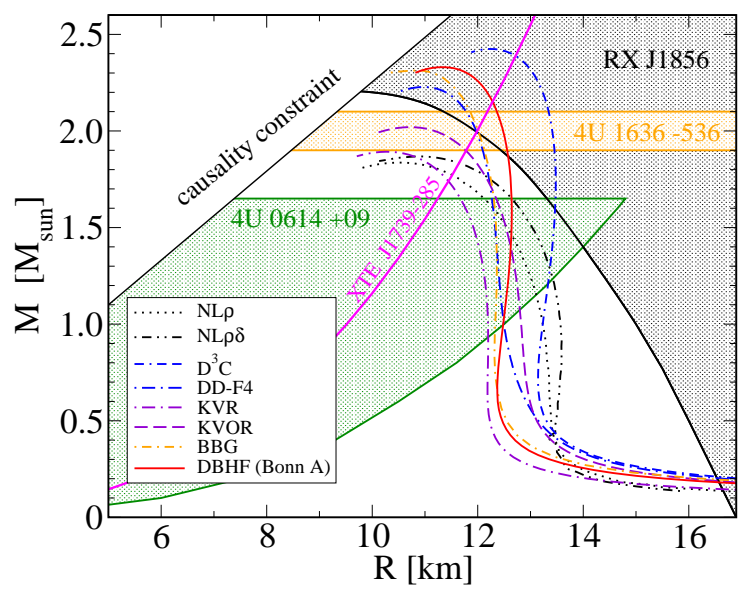

FIGURE 1. Mass-radius constraints from thermal radiation of the isolated NS RX J1856 (grey hatched region) and from QPOs in the LMXBs 4U 0614+09 (wedge-like green-hatched area) and 4U 1636-536 (orange hatched region). For 4U 1636-536 a mass of $2.0 \pm 0.1 \mathrm{M}_{\odot}$ is obtained so that the weak QPO constraint would exclude the NL $\rho$ and NL $\rho \delta$ EoS, whereas the strong one renders only the stiffest EoS D ${ }^{3}$ C, DD-F4, BBG and DBHF viable. XTE J1739-285 [17, 3] would favor a soft EoS, which is controversial, however (see text).

reproducible changes in QPO properties are related to the ISCO. If so, this implies that several NS in low-mass X-ray binaries should have gravitational masses between $1.9 \mathrm{M}_{\odot}$ and possibly $2.1 \mathrm{M}_{\odot}$ [16]. In Fig. 1] we show the estimated mass of $2.0 \pm 0.1 \mathrm{M}_{\odot}$ for $4 \mathrm{U} 1636-536$.

Recently, mass-radius constraints have been reported for the accreting compact stars XTE J1739-285 [18] and SAX J1808.4-3658 [19] (SAX J1808 for short) which are based on the identification of the burst oscillation frequency with the spin frequency of the compact star. It is almost impossible to fulfill the constraints from RX J1856 (and other highmass candidates) and SAX J1808 (which favors a soft EoS) simultaneously so that the status of SAX J1808 is currently controversial. It is likely that the small radius estimate of Leahy et al. [19] is a consequence of the underestimation of higher harmonics when only timing data are analyzed and not also the energy spectra.

The nearby isolated NS RX J1856.5-3754 (RX J1856 for short) belongs to a group of seven objects which show a purely thermal spectrum in X-rays and in optical-UV. This allows the determination of $R_{\infty} / d$, the ratio of the photospheric radius $R_{\infty}$ to the distance $d$ of the object, if the radiative properties of its photosphere are known. RX $\mathrm{J} 1856$ is the only object of this group which has a measured distance obtained by Hubble Space Telescope (HST) astrometry. After the distance of $117 \mathrm{pc}$ [20] became known several groups pointed out that the blackbody radius of this star is as large as 15 to $17 \mathrm{~km}$. Although both the X-ray and the optical-UV spectra are extremely well represented by blackbody functions they require different emission areas, a smaller hot spot and a larger cooler region. The overall spectrum could also be fitted by blackbody emission from a surface showing a continuous temperature distribution between a hot pole and a cool equator, as expected for a magnetized NS. The resulting blackbody radii are $17 \mathrm{~km}$ (two blackbodies) and $16.8 \mathrm{~km}$ (continuous temperature distribution) [21]. In this paper we adopt the result of the continuous temperature fit, $R_{\infty}=16.8 \mathrm{~km}$. More recent HST observations of RX J1856 indicated larger distances of up to $178 \mathrm{pc}$. A distance of around $140 \mathrm{pc}$ for RX J1856 is considered a conservative lower limit.For a distance of $140 \mathrm{pc}$ the corresponding radius is $17 \mathrm{~km}$ [22]. Although some questions-in particular that of the distance-are not yet finally settled, the recent data support an unusually large radius for RX J1856.5-3754.

\section{Elliptic Flow in Heavy Ion Collisions}

The flow of matter in heavy ion collisions is directed both forward and perpendicular (transverse) to the beam axis. At high densities spectator nucleons may shield the transversal flow into their direction and generate an inhomogeneous density and thus a pressure profile in the transversal plane. This effect is commonly referred to as elliptic flow and depends directly on the given EoS. An analysis of these nucleon flow data, which depends essentially only on the 

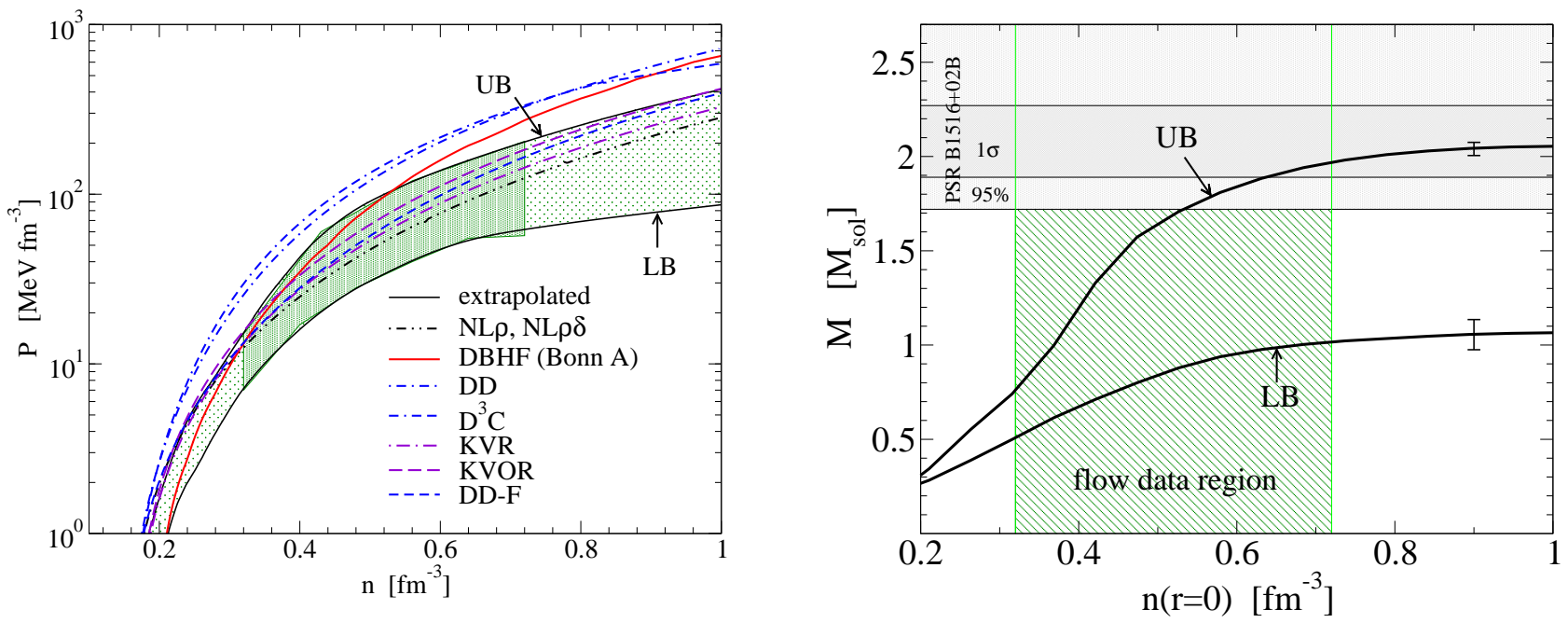

FIGURE 2. Left panel: Pressure region consistent with experimental flow data in symmetric nuclear matter (dark shaded region). The light shaded region extrapolates this region to higher densities within an upper (UB) and lower border (LB). Right panel: Mass versus central density for compact star configurations, calculated using the UB and LB extrapolations of the flow constraint boundaries. The error bars take into account different models for the symmetry energies. The gray horizontal bars denote the expected mass of PSR B1516+02B within the $1 \sigma$ interval and a likelihood of a mass below $1.72 \mathrm{M}_{\odot}$ of $5 \%$, resp. The vertical bars limit the density region covered by the flow constraint.

isospin independent part of the EoS, was carried out in a particular model in Ref. [23]. In particular it was determined for which range of parameters of the EoS the model is still compatible with the flow data. The region thus determined is shown in Fig. 2] as the dark shaded region. Ref. [23] then asserts that this region limits the range of accessible pressure values at a given density. For our purposes we extrapolated this region by an upper (UB) and lower (LB) boundary, enclosing the light shade region in Fig. 2

Thus the area of allowed values does not represent experimental values itself, but results from transport calculations for the motion of nucleons in a collision [23]. Of course, it would be preferable to use the actual flow data as a constraint for each specific EoS, but a corresponding testing tool based on a transport code is not yet available. Therefore we adopt the results of ref. [23] as a reasonable estimate of the preferable pressure-density domain in symmetric nuclear matter. Its upper boundary is expected to be stable against temperature variations [24]. The important fact is that the flow constraint probes essentially only the symmetric part of the energy per nucleon $E_{0}(n)$. Following Ref. [23] the constraint arises for a density window between 2 and 4.5 times saturation density $n_{s}$. One has, however, to keep in mind that at high densities this constraint is based on flow data from the AGS energy regime $\left(E_{\text {lab }} \sim 0.4-11\right.$ $\mathrm{AGeV})$. At these energies a large amount of the initial bombarding energy is converted into new degrees of freedom, i.e. excitations of higher lying baryon resonances and mesons, which makes conclusions on the nuclear EoS more ambiguous than at low energies. Nevertheless, the analysis of [23] provides a guideline for the high density regime which we believe to be reasonable. The uncertainties are represented by a region of possible pressures in Fig. 2.

An EoS which is in accordance with the flow constraint might still violate the maximum NS mass constraint. This is demonstrated in Fig. 2, where in particular the lower boundary of the limiting region in Fig. 2 was extrapolated to construct an artificial EoS (LB) in order to obtain the mass-density relation for corresponding compact star configurations. The resulting mass curve in Fig. 2 is far from reaching even typical NS mass values as the wellmeasured mass $M_{B 1913+16}=1.4408 \pm 0.0003 \mathrm{M}_{\odot}[$ [25]. This demonstrates that the lower bound (LB) in Fig. 2] does not agree with astrophysical observations, which therefore potentially narrow the band of the flow constraint.

The maximum mass constraint demands a certain stiffness of $E_{0}(n)$ in order to obtain sufficiently large maximum NS masses. The small influence of $E_{S}(n)$ on the NS mass can also be well recognized on Fig. 2. Two different symmetry energies, necessary to describe NS matter, were taken from the investigated EoS. They were chosen in accordance with the DU-constraint and gave the largest (DD-F) and smallest $\left(\mathrm{D}^{3} \mathrm{C}\right)$ contribution to the binding energy at $n=1 \mathrm{fm}^{-3}$. The resulting deviations of the NS masses are shown as error bars on the curves in Fig. 2 It results in a maximum difference of less than $0.2 \mathrm{M}_{\odot}$ for the mass curves corresponding to LB. The same was done for an artificial EoS extrapolating the upper boundary (UB). Here, the largest error estimate of approximately $0.1 \mathrm{M}_{\odot}$ is even smaller. The 
TABLE 1. A set of nuclear equations of state. The entries are: saturation density, $n_{s}$; binding energy, $a_{V}$; incompressibility, $K$; skewness parameter, $K^{\prime}$; symmetry energy, $J$; symmetry energy derivative, $L$; Dirac effective mass, $m_{D}$.

\begin{tabular}{l|llrrrrr} 
EoS & $n_{s}$ & $\begin{array}{l}a_{V} \\
{\left[\mathrm{fm}^{-3}\right]}\end{array}$ & $\begin{array}{r}K \\
{[\mathrm{MeV}]}\end{array}$ & $\begin{array}{r}K^{\prime} \\
{[\mathrm{MeV}]}\end{array}$ & $\begin{array}{r}J \\
{[\mathrm{MeV}]}\end{array}$ & $\begin{array}{r}L \\
{[\mathrm{MeV}]}\end{array}$ & $\begin{array}{r}m_{D} \\
{[\mathrm{MeV}]}\end{array}$ \\
\hline NL $\rho$ & 0.1459 & -16.062 & 203.3 & 576.5 & 30.8 & 83.1 & 0.603 \\
NL $\rho \delta$ & 0.1459 & -16.062 & 203.3 & 576.5 & 31.0 & 92.3 & 0.603 \\
$\mathrm{D}^{3} \mathrm{C}$ & 0.1510 & -15.981 & 232.5 & -716.8 & 31.9 & 59.3 & 0.541 \\
DD-F4 & 0.1469 & -16.028 & 220.4 & 1229.2 & 32.7 & 58.7 & 0.556 \\
KVR & 0.1600 & -15.800 & 250.0 & 528.8 & 28.8 & 55.8 & 0.805 \\
KVOR & 0.1600 & -16.000 & 275.0 & 422.8 & 32.9 & 73.6 & 0.800 \\
DBHF & 0.1810 & -16.150 & 230.0 & 507.9 & 34.4 & 69.4 & 0.678 \\
BBG & 0.1901 & -14.692 & 221.6 & -132.4 & 36.3 & 79.4 & - \\
DD-RH & 0.172 & -15.73 & 249.0 & - & 34.4 & 90.2 & 0.686
\end{tabular}

maximum mass for $\mathrm{UB}$ of about $2.0 \mathrm{M}_{\odot}$ again fulfills the corresponding constraint.

\section{NUCLEAR MATTER}

While modern nuclear matter models give a rather similar description of the saturation and sub-saturation behavior, they differ considerably in their extrapolations to densities above $\approx 2 n_{S}$, the regime which is relevant for NS physics and heavy ion collisions. This is illustrated in Table 1 , where several nuclear EoS are characterized by comparing the parameters of an expansion around saturation as a function of the density deviation $\varepsilon=\left(n-n_{s}\right) / n_{s}$ and the asymmetry $\beta$ according to

$$
E=a_{V}+\frac{K}{18} \varepsilon^{2}-\frac{K^{\prime}}{162} \varepsilon^{3}+\ldots+\beta^{2}\left(J+\frac{L}{3} \varepsilon+\ldots\right) .
$$

Most of the shown models are derived in the framework of the relativistic mean-field approach, [26, 27, 28, 29] allowing for non-linear (NL) self-interactions of the $\sigma$ meson [30]. For model NL $\rho$, the isovector part of the interaction is described entirely in terms of $\rho$ meson exchange. This is different for NL $\rho \delta$ where the isovector part of the interaction is described in terms of both $\rho$ and $\delta$-meson exchange. The latter is generally neglected in RMF models [31]. RMF models with density dependent input parameters (coupling constants and masses) are represented in Table 1 by four different models from two classes, where in the first one density dependent meson couplings are modeled such that several properties of finite nuclei (binding energies, charge and diffraction radii, surface thicknesses, neutron skin in ${ }^{208} \mathrm{~Pb}$, spin-orbit splittings) can be fitted [32]. $\mathrm{D}^{3} \mathrm{C}$ additionally contains a derivative coupling which leads to momentum-dependent nucleon self-energies, and DD-F4 is modeled such that the flow constraint from heavy ion collisions is fulfilled [23]. The second class of these models is motivated by the Brown-Rho scaling assumption [33] that not only the nucleon mass but also the meson masses should decrease with increasing density. In the KVR and KVOR models [34] these dependences are related to a nonlinear scaling function of the $\sigma$-meson field such that the EoS of symmetric nuclear matter and pure neutron matter below four times the saturation density coincide with those of the Urbana-Argonne group [35]. In this way the latter approach builds a bridge between the phenomenological RMF models and a microscopic EoS built on realistic nucleon-nucleon forces. The RMF models are contrasted with several variational models for the EoS such as APR [35], WFF[36], FPS[37], a relativistic Dirac-Brueckner-Hartree-Fock (DBHF) model [38, 39], and a nonrelativistic Brueckner-Bethe-Goldstone (BBG) model [40].

As shown in Fig. 3 none of these EoS predicts a maximum NS mass below the $2 \sigma$ mass limit of $1.68 \mathrm{M}_{\odot}$ for PSR $\mathrm{B} 1516+02 \mathrm{~B}$, and even at the $1 \sigma$ mass limit of $1.84 \mathrm{M}_{\odot}$ the softest EoS NL $\rho$ and NL $\rho \delta$ cannot be excluded. This underlines the important rule of the maximum NS mass as a constraint on the high density EoS. If a pulsar with a mass exceeding $1.8-1.9 \mathrm{M}_{\odot}$ at the $2 \sigma$ or even $3 \sigma$ level would be observed in the future, this will impose a severe constraint on the stiffness of the nuclear EoS. For the set of EoS tested here, only the stiffest models, i.e. $\mathrm{D}^{3} \mathrm{C}$, DD-F4, BBG, and DBHF would remain viable candidates. It is noteworthy, that the flow constraint limits the stiffness of the EoS to be 'rather' soft, whereas the higher the constraining maximum NS mass is the 'stiffer' the EoS needs to be. Both together provide complementing data. 


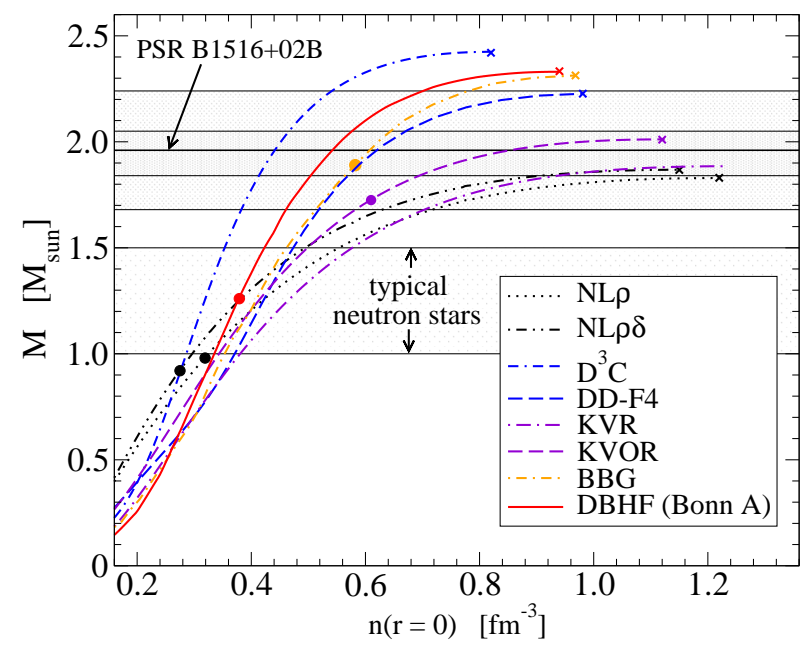

FIGURE 3. Mass versus central density of NS, computed for the EoS shown in Table 1 Crosses refer to the maximum-mass star of each sequence, filled dots mark the critical masses and central densities beyond which the direct Urca (DU) cooling process becomes possible.

\section{QUARK MATTER}

\section{Nonperturbative QCD}

Confinement and dynamical chiral symmetry breaking (DCSB) are key emergent phenomena in QCD. Neither of these is apparent in QCD's Lagrangian and yet they play a dominant role in determining the observable characteristics of real-world QCD. A natural starting point for an exploration of DCSB is QCD's gap equation.

$$
S(p ; \mu)^{-1}=Z_{2}\left(i \vec{\gamma} \cdot \vec{p}+i \gamma_{4}\left(p_{4}+i \mu\right)+m^{\mathrm{bm}}\right)+\Sigma(p ; \mu),
$$

with the renormalized self energy expressed as

$$
\Sigma(p ; \mu)=Z_{1} \int_{q}^{\Lambda} g^{2}(\mu) D_{\rho \sigma}(p-q ; \mu) \frac{\lambda^{a}}{2} \gamma_{\rho} S(q ; \mu) \Gamma_{\sigma}^{a}(q, p ; \mu),
$$

where $\int_{q}^{\Lambda}$ represents a translationally invariant regularization of the integral, with $\Lambda$ the regularization mass-scale, $D_{\rho \sigma}(k ; \mu)$ is the dressed-gluon propagator, $\Gamma_{\sigma}^{a}(q, p ; \mu)$ is the dressed-quark-gluon vertex, and $m^{\text {bm }}$ is the $\Lambda$-dependent current-quark bare mass. The quark-gluon-vertex and quark wave function renormalization constants, $Z_{1,2}\left(\zeta^{2}, \Lambda^{2}\right)$, depend on the renormalization point, $\zeta$, the regularization mass-scale and the gauge parameter. A nonzero chemical potential introduces no divergences in addition to those present in the $\mu=0$ theory. Hence, the renormalization constants determined at $\mu=0$ are completely sufficient in-medium. The gap equation's solution can assume the general form [41]

$$
S(p ; \mu)^{-1}=i \vec{\gamma} \cdot \vec{p} A\left(p^{2}, p \cdot u, \zeta^{2}\right)+i \gamma_{4}\left(p_{4}+i \mu\right) C\left(p^{2}, p \cdot u, \zeta^{2}\right)+B\left(p^{2}, p \cdot u, \zeta^{2}\right),
$$

where $u=(\overrightarrow{0}, i \mu)$. The mass function $M\left(p^{2}, p \cdot u\right)=B\left(p^{2}, p \cdot u, \zeta^{2}\right) / A\left(p^{2}, p \cdot u, \zeta^{2}\right)$ is renormalization point independent. There are numerous studies of this basic Dyson-Schwinger equation in-vacuum [42, 43] and in-medium [44]. It is noteworthy that extant self-consistent studies of concrete models of QCD, which exhibit both confinement and DCSB in vacuum, possess coincident deconfinement and chiral symmetry restoration transitions; e.g., Refs. [45, 46, 47, 48, 49]. This result appears to follow from the crucial role played by the in-medium evolution of the dressed-quark self-energy in both transitions.

Significant effort continues to be expended on determining the precise nature of the kernel of QCD's gap equation. A dialogue between DSE studies and results from numerical simulations of lattice-regularized QCD is providing 

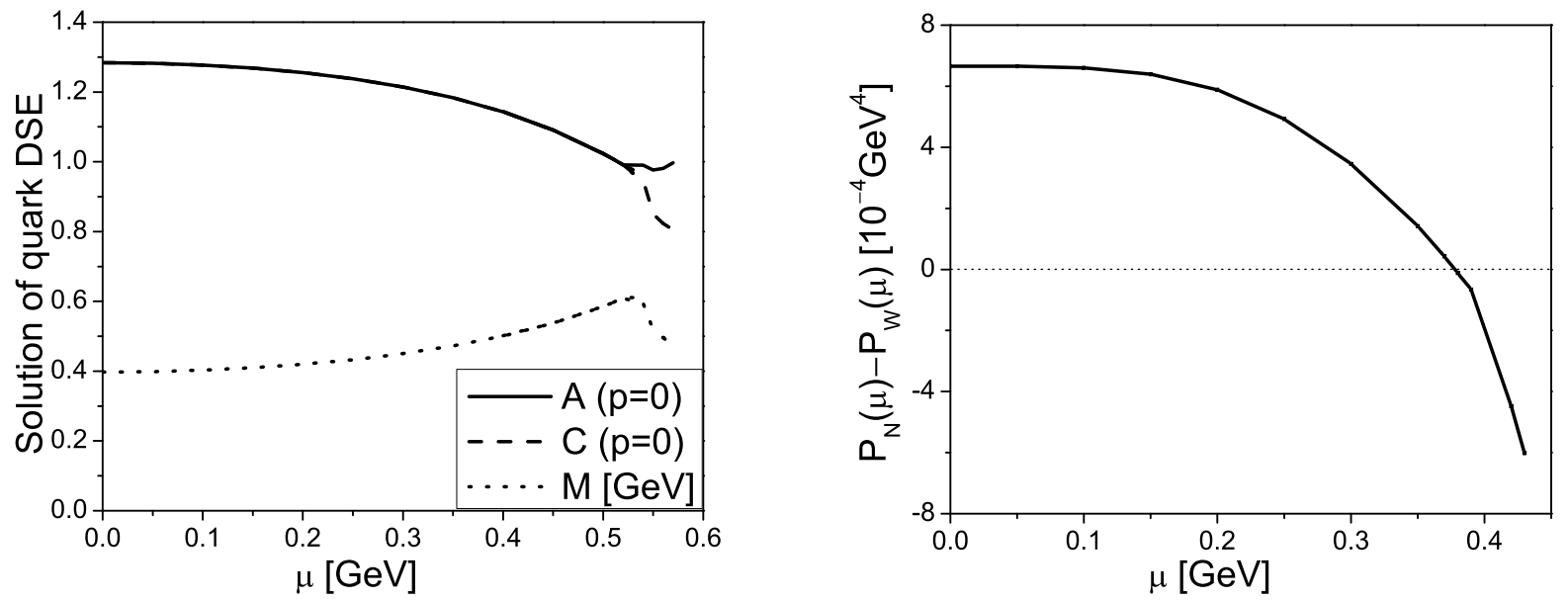

FIGURE 4. Left panel: Evolution with $\mu$ of the dimensionless quantities $A(p=0 ; \mu), C(p=0 ; \mu)$, and $M(p=0 ; \mu)$ measured in $\mathrm{GeV}$, computed from the gap equation (4). Right Panel: Difference in pressure between the Nambu-Goldstone and Wigner solutions of the gap equation. Observe, that the Wigner phase is realized for $\mu>0.38 \mathrm{GeV}$, which results in a first order phase DCSB transition at this value.

important information; e.g., Refs. [50, 51, 52, 53, 54, 55, 56, 57, 58]. This body of work can be used to formulate reasonable Ansätze for the dressed-gluon propagator and dressed-quark-gluon vertex in Eq. (4).

DCSB is signaled by the appearance of a gap equation solution in which the Dirac-scalar piece is nonzero in the chiral limit. This effect owes primarily to a dense cloud of gluons that clothes a low-momentum quark [59, 60]. DCSB is the single most important mass generating mechanism for light-quark hadrons; e.g., one can identify it as being responsible for roughly $98 \%$ of a proton's mass. A system in which the gap equation's DCSB solution is the favored ground state is said to realize chiral symmetry in the Nambu-Goldstone mode. The antithesis is termed a Wigner-Weyl realization of the symmetry. Since a system's ground state is that configuration for which the pressure is a global maximum the pressure difference between the Nambu-Goldstone and Wigner phase reveals which of both is realized. A striking signature of dynamical chiral symmetry breaking is a nonzero value of the dressed-quark mass function $M(p, \mu)$, which entails a nonzero chiral-limit quark condensate. Naturally, a nonzero chemical potential destabilizes the quark condensate and, with increasing chemical potential, results in a first-order chiral symmetry restoring transition at $\mu \approx M(0)$. This is illustrated in Fig. 4. Evidence suggests a coincident first-order deconfinement transition [5].

\section{Effective Quark Matter Models}

There is no inherent obstacle that prohibits an extension of the powerful Dyson-Schwinger approach to the finite density domain and in fact it appears as the most promising candidate for a future systematic modeling of dense QCD matter in compact stars [44, 46, 61]. However, for explorative studies on the possibly rich phase structure of dense matter in QCD it is for practical reasons useful to employ effective models which preserve certain but not necessarily all features of QCD. Increasingly applied are models of the Nambu-Jona-Lasinio (NJL) type where chiral symmetry is dynamically broken in the nonperturbative vacuum and restored at finite densities and temperatures. Although the standard NJL model is quite simple, based on a few parameters, such as momentum cutoff and coupling constants for a set of interaction channels, it offers possibilities for generalizations, like a density-dependence of the cutoff parameter [62], momentum-dependent formfactors [63] and an infrared cutoff to mimic confinement [64, 65].

Essentially one starts from a $Q C D$-motivated partition function

$$
Z(T, \hat{\mu})=\int \mathscr{D} \bar{q} \mathscr{D} q \exp \left\{\int_{0}^{\beta} d \tau \int d^{3} x\left[\bar{q}\left(i \not \partial-\hat{m}_{0}+\hat{\mu} \gamma^{0}\right) q+\mathscr{L}_{\text {int }}\right]\right\}
$$




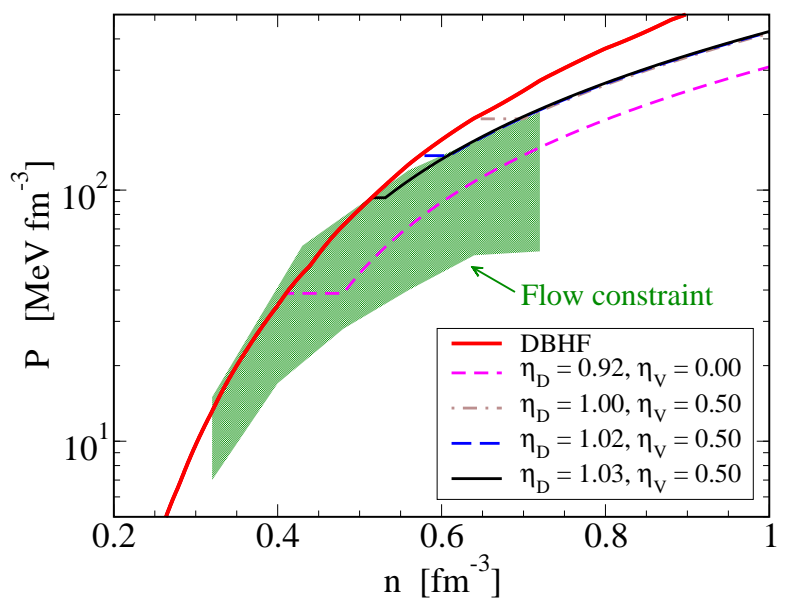

FIGURE 5. Hybrid EoS with a low density hadronic branch described by the DBHF approach and a high density quark matter branch obtained from a three-flavor NJL model with color superconductivity (diquark coupling $\eta_{D}$ ) and isoscalar vector meson coupling $\left(\eta_{V}\right)$.

which explicitly accounts for phases of possible interest by considering specific channels in the interaction part $\mathscr{L}_{\text {int }}$ of the Lagrangian. Mass gaps, pairing gaps and the EoS are then obtained from the mean-field thermodynamic potential, $\Omega_{M F}=-T \ln Z_{M F}$. As example serves the following model [66] with diquark pairing in the single flavor color-spinlocking (CSL), two-flavor (2SC) and three-flavor color-flavor locking (CFL) channels,

$$
\mathscr{L}_{\text {int }}=G_{S}\left\{\sum_{a=0}^{8}\left[\left(\bar{q} \tau_{a} q\right)^{2}+\left(\bar{q} i \gamma_{5} \tau_{a} q\right)^{2}\right]+\eta_{D 0} \sum_{A=2,5,7} j_{D 0, A}^{\dagger} j_{D 0, A}+\eta_{D 1} j_{D 1}^{\dagger} j_{D 1}\right\},
$$

where $\hat{\mu}=\frac{1}{3} \mu_{B}+\operatorname{diag}_{f}\left(\frac{2}{3},-\frac{1}{3},-\frac{1}{3}\right) \mu_{Q}+\lambda_{3} \mu_{3}+\lambda_{8} \mu_{8}$ is the diagonal quark chemical potential matrix and $\hat{m}_{0}=$ $\operatorname{diag}_{f}\left(m_{u}^{0}, m_{d}^{0}, m_{s}^{0}\right)$ the current-quark mass matrix. The relative coupling strengths of the spin-0 and spin-1 diquark currents, $j_{D 0, A}=q^{T} i C \gamma_{5} \tau_{A} \lambda_{A} q$ and $j_{D 1}=q^{T} i C\left(\gamma_{1} \lambda_{7}+\gamma_{2} \lambda_{5}+\gamma_{3} \lambda_{2}\right) q$, are essentially free parameters, but can be chosen according to a Fierz transformation of the one-gluon exchange interaction, $\eta_{D 0}=3 / 4$ and $\eta_{D 1}=3 / 8$, see [67]. We point out, that bosonised diquark fields in QCD play an essential role in the color singlet baryon states [68].

The next section shows how observational constraints help to fix a model EoS obtained in this framework.

\section{THE QUARK HADRON PHASE TRANSITION}

A strategy to construct hybrid EoS in agreement with observational data is to adjust available coupling strengths within certain limits to a subset of available data. This is demonstrated in Fig 5 for a model with an interaction different from that in Eq. (7). Here the coupling strength of the scalar diquark $\left(\eta_{D}\right)$ and isoscalar vector meson $\left(\eta_{V}\right)$ channel are considered as basically free parameters and have been adjusted so, that after constructing the (Maxwell-)phase transition from the nuclear DBHF EoS to this QM EoS the hybrid EoS fits the upper limit of the flow constraint [69]. Just by adjusting to this requirement the hybrid EoS fulfills all recently developed constraints from modern compact star observations [2]. It is an interesting and encouraging result, that none of the present constraints on the mass-radius relation of NS rules out hybrid stars [70, 6].

As a last point we discuss ambiguities in the way the phase transition is modeled. The $\beta$-equilibrium condition in compact stars relates the chemical potentials of quarks and electrons by $\mu_{d}=\mu_{s}$ and $\mu_{d}=\mu_{u}+\mu_{e}$. The mass difference between the strange and the light quark flavors $m_{s}>m_{u}, m_{d}$ induces different densities of the down and strange quark. Charge neutrality requires a finite electron density and consequently $\mu_{d}>\mu_{u}$. When increasing the baryochemical potential, the d-quark chemical potential is the first to reach a critical value where the the partial density of free d-quarks becomes finite in a first order phase transition. Due to the finite value of $\mu_{e}$ the u-quark chemical potential is below the critical value and the s-quark density is zero due to the high s-quark mass. Therefore the negative electric charge of 


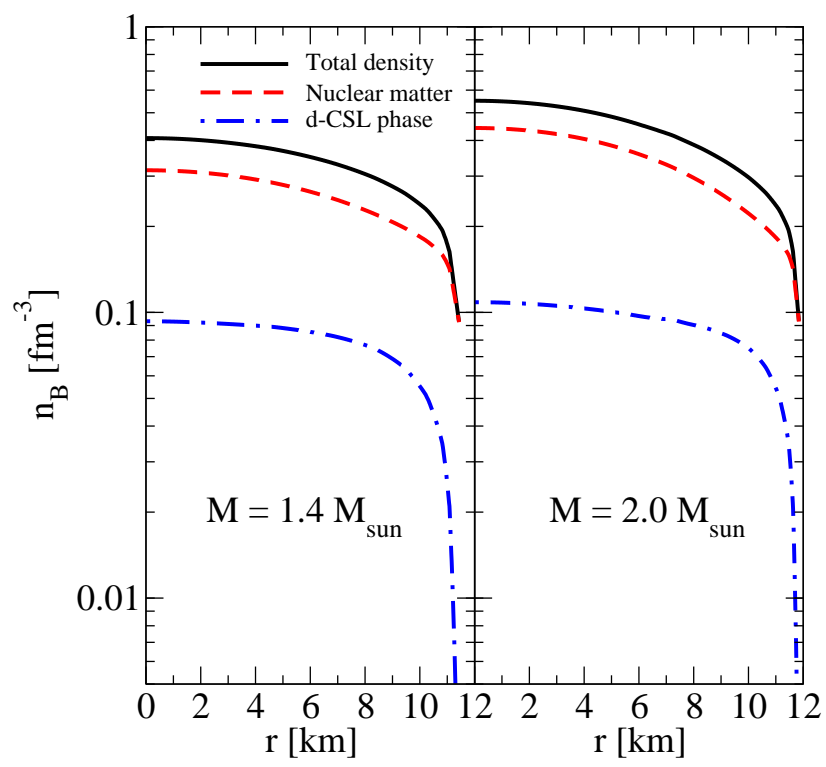

FIGURE 6. Density profiles of two stars with masses $1.4 \mathrm{M}_{\odot}$ and $2.0 \mathrm{M}_{\odot}$. In this model a mixed phase of d-CSL quark matter with nuclear matter extends up to the crust-core boundary.

the d-quark cannot be compensated in a pure quark phase and single-flavor d-quark matter under NS conditions does not exit. The situation changes considering a chemical equilibrium of the type $n+n \leftrightarrow p+3 d$, with positive charged protons which compensate the charge of a single-flavor d-quark phase and results in a mixed phase of nucleons and down quarks once the d-quark chemical potential exceeds the critical value. This can be thought analogous to the dissociation of nuclear clusters in the crust of NS (neutron dripline). One interesting consequence of the effect is illustrated in Fig 6 Ignoring in a first approach important effects of the inhomogeneous phase mixture, e.g. Coulomb interactions and surface tension, the existence of such a phase suggests small fractions of quark matter even near the the crust-core boundary. We point out, that neither way to model the phase transition can be considered to be "correct". Nucleons actually consist of quarks and as expressed before it will take serious efforts to describe the QCD phase transition within an appropriate and fundamental approach.

\section{ACKNOWLEDGMENTS}

We acknowledge useful communications with and the support of E. F. Brown, H. Chen, I.C. Cloët, P. Danielewicz, Y.X. Liu, M.C. Miller, and R. Rutledge. We are grateful to the organizers of the Fifth ANL/INT/MSU/JINA FRIB Theory Workshop. This work was supported by: the Department of Energy, Office of Nuclear Physics, contract no. DE-AC0206CH11357 (TK,CDR); the Belgian fund for scientific research FNRS (FS); the Polish Ministry for Research and Higher Education, grant no. N N 202095333 (DBB); CompStar, an ESF Research Networking Programme (DBB,FS).

\section{REFERENCES}

1. D. Blaschke, T. Klahn, and D. N. Voskresensky, Astrophys. J. 533, 406-412 (2000), astro-ph/9908334

2. T. Klahn, et al., Phys. Rev. C74, 035802 (2006), nucl-th/0602038.

3. J. M. Lattimer, and M. Prakash, Phys. Rept. 442, 109-165 (2007), astro-ph/0612440.

4. D. Blaschke, T. Klahn, and F. Weber (2008), 0808.1279.

5. H. Chen, et al., Phys. Rev. D78, 116015 (2008), 0807.2755

6. T. Klahn, et al., Phys. Lett. B654, 170-176 (2007), nucl-th/0609067.

7. D. Blaschke, F. Sandin, T. Klahn, and J. Berdermann (2008), 0807.0414

8. D. J. Champion, et al. (2008), 0805.2396

9. P. C. C. Freire, A. Wolszczan, M. v. d. Berg, and J. W. T. Hessels (2007), 0712.3826

10. S. E. Thorsett, and D. Chakrabarty, Astrophys. J. 512, 288 (1999), astro-ph/9803260 
11. M. van der Klis, Ann. Rev. Astron. Astrophys. 38, 717-760 (2000), astro-ph/ 0001167

12. M. C. Miller, AIP Conf. Proc. 714, 365-370 (2004), astro-ph/0312449.

13. M. C. Miller, F. K. Lamb, and D. Psaltis, Astrophys. J. 508, 791-830 (1998), astro-ph/9609157

14. S. van Straaten, E. C. Ford, M. van der Klis, M. Mendez, and P. Kaaret, Astrophys. J. 540, 1049 (2000), astro-ph/0001480.

15. W. Zhang, A. P. Smale, T. E. Strohmayer, and J. H. Swank, Astrophys. J. 503, L147 (1998), ast ro-ph/9804228

16. D. Barret, J.-F. Olive, and M. C. Miller, Mon. Not. Roy. Astron. Soc. 361, 855-860 (2005), ast ro-ph/05054 02.

17. P. Kaaret, et al. (2006), astro-ph/0611716

18. G. Lavagetto, I. Bombaci, A. D’Ai', I. Vidana, and N. R. Robba (2006), astro-ph/0612061.

19. D. A. Leahy, S. M. Morsink, and C. Cadeau, Astrophys. J. 672, 1119 (2008), astro-ph/0703287

20. F. M. Walter, and J. Lattimer, Astrophys. J. 576, L145-L148 (2002), a st ro-ph/ 0204199.

21. J. E. Trumper, V. Burwitz, F. Haberl, and V. E. Zavlin, Nucl. Phys. Proc. Suppl. 132, 560-565 (2004), a stro-ph/0312600.

22. W. Ho, "Proc. of the Int. Conf. on Isolated Neutron Stars: From the Interior to the Surface," in Astrophys. Space Sci., London, England, 2006.

23. P. Danielewicz, R. Lacey, and W. G. Lynch, Science 298, 1592-1596 (2002), nucl-th/0208016

24. P. Danielewicz, Private communication (2006).

25. I. Stairs, Science 304, 547 (2004).

26. J. Walecka, Ann. Phys. (N.Y.) 83, 491 (1974).

27. B. Serot, and J. Walecka, Adv. Nucl. Phys. 16, 1 (1986).

28. P. G. Reinhard, Rep. Prog. Phys. 52, 439 (1989).

29. P. Ring, Prog. Part. Nucl. Phys. 37, 193 (1996).

30. T. Gaitanos, et al., Nucl. Phys. A732, 24-48 (2004), nucl-th/0309021

31. B. Liu, V. Greco, V. Baran, M. Colonna, and M. Di Toro, Phys. Rev. C65, 045201 (2002), nucl-th/0112034

32. S. Typel, Phys. Rev. C71, 064301 (2005), nucl-th/0501056

33. G. E. Brown, and M. Rho, Phys. Rev. Lett. 66, 2720-2723 (1991).

34. E. E. Kolomeitsev, and D. N. Voskresensky, Nucl. Phys. A759, 373-413 (2005), nucl-th/ 0410063

35. A. Akmal, V. R. Pandharipande, and D. G. Ravenhall, Phys. Rev. C58, 1804-1828 (1998), nucl-th/9804027

36. R. B. Wiringa, V. Fiks, and A. Fabrocini, Phys. Rev. C38, 1010-1037 (1988).

37. B. Friedman, and V. R. Pandharipande, Nucl. Phys. A361, 502-520 (1981).

38. E. N. E. van Dalen, C. Fuchs, and A. Faessler, Nucl. Phys. A744, 227-248 (2004), nucl-th/ 0407070

39. E. N. E. van Dalen, C. Fuchs, and A. Faessler, Phys. Rev. Lett. 95, 022302 (2005), nucl-th/ 0502064

40. M. Baldo, G. F. Burgio, and H. J. Schulze, Phys. Rev. C61, 055801 (2000), nucl-th/9912066

41. J. J. Rusnak, and R. J. Furnstahl, Z. Phys. A352, 345-350 (1995).

42. P. Maris, and C. D. Roberts, Int. J. Mod. Phys. E12, 297-365 (2003), nucl-th/0301049.

43. C. D. Roberts, M. S. Bhagwat, A. Holl, and S. V. Wright, Eur. Phys. J. ST 140, 53-116 (2007), 0802.0217

44. C. D. Roberts, and S. M. Schmidt, Prog. Part. Nucl. Phys. 45, S1-S103 (2000), nucl-th/ 0005064

45. A. Bender, D. Blaschke, Y. Kalinovsky, and C. D. Roberts, Phys. Rev. Lett. 77, 3724-3727 (1996), nucl-th/9606006

46. D. Blaschke, C. D. Roberts, and S. M. Schmidt, Phys. Lett. B425, 232-238 (1998), nucl-th/9706070

47. A. Bender, G. I. Poulis, C. D. Roberts, S. M. Schmidt, and A. W. Thomas, Phys. Lett. B431, 263-269 (1998), nucl-th/9710069

48. P. Maris, and P. C. Tandy (2001), nucl-th/0109035

49. A. Bashir, A. Raya, I. C. Cloet, and C. D. Roberts, Phys. Rev. C78, 055201 (2008), 0806.3305

50. M. S. Bhagwat, M. A. Pichowsky, C. D. Roberts, and P. C. Tandy, Phys. Rev. C68, 015203 (2003), nucl-th/0304003.

51. R. Alkofer, W. Detmold, C. S. Fischer, and P. Maris, Phys. Rev. D70, 014014 (2004), hep-ph/030 9077

52. M. S. Bhagwat, A. Holl, A. Krassnigg, C. D. Roberts, and P. C. Tandy, Phys. Rev. C70, 035205 (2004), nucl-th/ 0403012.

53. M. S. Bhagwat, and P. C. Tandy, Phys. Rev. D70, 094039 (2004), hep-ph/0407163

54. M. S. Bhagwat, and P. C. Tandy, AIP Conf. Proc. 842, 225-227 (2006), nucl-th/ 0601020

55. A. Kizilersu, D. B. Leinweber, J.-I. Skullerud, and A. G. Williams, Eur. Phys. J. C50, 871-875 (2007), hep-lat/0610078

56. W. Kamleh, P. O. Bowman, D. B. Leinweber, A. G. Williams, and J. Zhang, Phys. Rev. D76, 094501 (2007), 0705.4129

57. P. Boucaud, et al. (2008), 0803.2161.

58. A. Cucchieri, and T. Mendes (2008), 0804.2371

59. M. S. Bhagwat, I. C. Cloet, and C. D. Roberts (2007), 0710.2059 .

60. C. D. Roberts, Prog. Part. Nucl. Phys. 61, 50-65 (2008), 0712.0633

61. D. Nickel, R. Alkofer, and J. Wambach, Phys. Rev. D74, 114015 (2006), hep-ph/ 0609198.

62. M. Baldo, G. F. Burgio, P. Castorina, S. Plumari, and D. Zappala, Phys. Rev. C75, 035804 (2007), hep-ph/0607343

63. S. M. Schmidt, D. Blaschke, and Y. L. Kalinovsky, Phys. Rev. C50, 435-446 (1994).

64. D. Blaschke, G. Burau, M. K. Volkov, and V. L. Yudichev, Phys. Atom. Nucl. 62, 1919-1923 (1999).

65. S. Lawley, W. Bentz, and A. W. Thomas, J. Phys. G32, 667-680 (2006), nucl-th/ 0602014.

66. D. Blaschke, F. Sandin, and T. Klahn, J. Phys. G35, 104077 (2008), 0808.0181

67. D. N. Aguilera, D. Blaschke, M. Buballa, and V. L. Yudichev, Phys. Rev. D72, 034008 (2005), hep-ph/0503288

68. R. T. Cahill, J. Praschifka, and C. Burden, Austral. J. Phys. 42, 161-169 (1989).

69. D. Blaschke, T. Klahn, and F. Sandin, J. Phys. G35, 014051 (2008), 0708.4216

70. M. Alford, et al., Nature 445, E7-E8 (2007), astro-ph/ 0606524 